\title{
Ungenutzte Potenziale aufdecken
}

\section{Stoff- und energieflussorientierte Kostenrechnungen stellen auch für landwirt- schaftliche Unternehmen ein systematisches Instrument zur Aufdeckung ökono- misch-ökologischer Verbesserungspotenziale dar. Die Notwendigkeit, das Vor- gehen und der Nutzen der Implementierung werden im Folgenden am Beispiel eines mittelständischen Agrarunternehmens erläutert.}

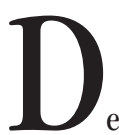

Von Matthias Kramer, Hans-Christian Brauweiler und Peggy Eifler en Ausgangspunkt für die folgenden Ausführungen bildet das von der Deutschen Bundesstiftung Umwelt geförderte anwendungsorientierte Projekt "Implementierung der Umweltkostenrechnung in kleinen und mittelständischen Unternehmen repräsentativer Branchen". Dieses bearbeitet der Studiengang BWL des IHI Zittau in Kooperation mit dem IÖB der Universität Siegen. Die stoff- und energieflussorientierte Kostenrechnung (SEFR) kann durch die systematische Aufdeckung von stoff- und energieflussbezogenen Optimierungspotenzialen das Umweltmanagement am besten unterstiitzen. Dies ist ein Ergebnis der Analyse existierender Umweltkostenrechnungsansätze aus Theorie und Praxis (vgl. dazu auch den Beitrag von Loew in diesem Heft). Nach dem Ansatz der SEFR umfassen Flusskosten alle Kosten für Güter und Dienste, die mit betrieblichen Stoff- und Energieflüssen verbunden sind, wobei davon ausgegangen wird, dass alle betrieblichen Material- und Energieströme umweltrelevant sind und somit umweltbezogene Kosten darstellen. Da die SEFR in der Praxis bislang noch selten Anwendung findet und sich für kleine und mittelständische Unternehmen sehr arbeitsaufwändig gestaltet, soll im Rahmen des Projekts die SEFR für den Einsatz in KMU weiter entwickelt werden.

\section{Relevanz der Landwirtschaft}

Für die praktische Erprobung wurden mehrere Unternehmen aus dem Produzierenden Gewerbe sowie ein landwirtschaftlicher Betrieb ausgewählt (1). Aufgrund der Intensivierung der landwirtschaftlichen Produktion nimmt nicht nur der Energie- und Materialverbrauch in der Landwirtschaft kontinuierlich zu, sondern auch die Belastung des Grundwassers und der Oberflächengewässer. Für die Berücksichtigung der Landwirtschaft spricht zudem, dass sie zuletzt durch die lange Dürreperiode im Jahr 2000 und die BSE- und MKS-Debatten im Frühjahr 2001 Gewinneinbußen verzeichnen musste. Von diesen blieb auch die im Folgenden näher betrachtete Agrargenossenschaft Bertsdorf-Olbersdorf e.G. nicht verschont. Die Agrargenossenschaft bewirtschaftet derzeit mit 33 Mitarbeitern eine Nutzfläche von 1.180 Hektar.

\section{Analyse der betrieblichen Situation}

Den umfassendsten Teil der Unternehmensanalyse bildete die Erhebung und Systematisierung der Stoff- und Energieströme in betrieblichen und prozessbezogenen Stoff- und Energiebilanzen nach dem Top-Down-Verfahren (2) mit folgenden Arbeitsschritten:

Qualitative Erfassung und Systematisierung umweltrelevanter Stoff- und Energieströme (verwendete Instrumente: Betriebliche Umweltbilanzen, Materialfließbilder),

Erhebung, Schätzung und Berechnung der Mengen und Kosten der Materialien,

- Gegenüberstellung der erhobenen Mengenund Kostendaten in betrieblichen sowie prozessbezogenen Stoff- und Energiebilanzen.

\section{Identifizierte Optimierungspotenziale}

Die betriebliche Umweltbilanz sowie die Prozessbilanzen bildeten die Basis für eine erste ökonomisch-ökologische Analyse, aus welcher Vorschläge für mögliche Optimierungsmaßnahmen abgeleitet wurden. So umfassen beispielsweise allein die Kosten für die beiden ökologisch sehr bedenklichen Produktionsmittel Mineraldünger und Pflanzenschutzmittel fast 20 Prozent der betrieblichen Materialkosten. Zudem ist zu beachten: Sowohl bei der Anwendung als auch bei der Bereitstellung (Produktion und Transport) dieser Chemikalien wird die Umwelt belastet. Aus Sicht der Agrargenossenschaft ist vor allem der Einsatz der Mittel im Unternehmen Ansatzpunkt und Beein-
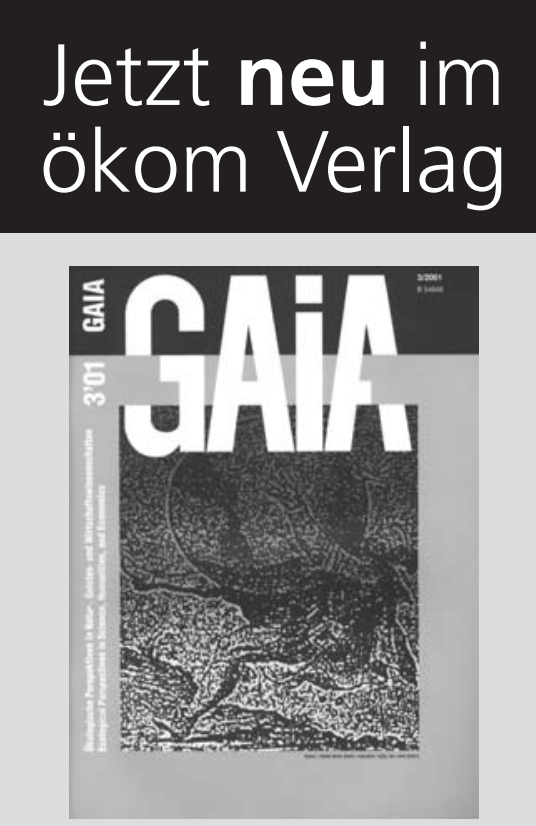

Hat die heutige Umweltforschung die Verbindung zur Umweltpolitik aus den Augen verloren? Oder hat die Politik falsche Erwartungen an die Umweltforschung gestellt? - essenzielle Fragestellungen der neu im ökom Verlag erscheinenden Zeitschrift GAIA. Das transdisziplinäre Medium für ökologische Perspektiven in Natur-, Geistes- und Wirtschaftswissenschaften setzt sich mit Umweltforschung in seiner ganzen Breite auseinander.

Lesen Sie in der aktuellen Ausgabe - 10 Jahre „Verhinderung eines amtlichen

BSE-Nachweises durch staatliche Stellen"

Wozu Umweltforschung? Teil II: Zum Leitbild "Reflexive Umweltforschung"

- Jenseits von Natur, Herrschaft und Geschlecht? Vier kritische Thesen zu Politik, Nachhaltigkeit und Wissenschaft

Partitioning of anthropogenic carbon dioxide The fragile role of carbon sinks

Vier Ausgaben jährlich im Normalabonnement für $75 € / 146,69 \mathrm{DM}$, Einzelheft für $19 € / 37,16$ DM erhältlich beim

ökom Verlag, Waltherstr. 29,

D - 80337 München,

Fon $++49 /(0) 89 / 544184-0$,

Fax $++49 /(0) 89 / 544184-49$,

kontakt@oekom.de,

www.oekom.de

Bestellen Sie ein kostenloses Probeheft! 
flussungsmöglichkeit für betriebliche Kosten- und Umweltentlastungen. Da die Herbizide unter den Pflanzenschutzmitteln einen beachtlichen Mengenanteil von rund 75 Prozent einnehmen, ist beispielsweise zu prüfen, ob einzelne Herbizide wirklich erforderlich sind oder ob nicht mit alternativen Maßnahmen der Entstehung der Unkräuter vorgebeugt bzw. deren Beseitigung umwelt- und kostenschonender durchgeführt werden kann.

Weitere Ansatzpunkte werden in der Anpassung der Fruchtfolgeauswahl und in der Optimierung der Verfahren bei der Ausbringung der Dünger und Pflanzenschutzmittel gesehen. Zwei weitere Verbesserungsvorschläge betreffen die interne Verwertung des in der Agrargenossenschaft kostenneutral anfallenden Holzes und eventuell auch

\section{Integrierte Technik integrieren?}

\section{Seit über 25 Jahren werden in Deutschland Umweltbelastungen und Umwelt- schutzkosten statistisch erfasst. Letzteres ist allerdings methodisch nicht un- problematisch. Deshalb werden seit fünf Jahren nur noch End-of-Pipe-Umwelt- schutzinvestitionen erhoben. Die EU möchte dies nun allerdings ändern.}

$\mathrm{U}$ Von Bernd Becker mweltstatistiken basieren auf Gesetzen, die von Zeit zu Zeit an neue Anforderungen angepasst werden. Das deutsche Umweltstatistikgesetz von 1994 hat im Vergleich zur Fassung von 1974 durch die Einführung mehrerer zusätzlicher und die Modifizierung bestehender Erhebungen das umweltpolitische Informationsangebot verbessert. Gleichzeitig war man 1994 bestrebt, die Betriebe der gewerblichen Wirtschaft zu entlasten, indem man vor allem die statistische Nutzung von umweltrelevanten Verwaltungsunterlagen ermöglichte.

Im Rahmen des jetzigen Umweltstatistikgesetzes werden zwei Typen von umweltpolitischen Merkmalen erhoben:

- Feststellung der Umweltbelastungen durch Emissionen, und zwar in den Bereichen Abfall, Wasser und Luft.

- Quantifizierung der ökonomischen Bedeutung des Umweltschutzes.

Insgesamt werden in den Bereichen Abfallwirtschaft, Wasserwirtschaft, Luftreinhaltung und Umweltökonomie mehr als 30 umweltstatistische Erhebungen durchgeführt (vgl. Tab. 1), die im Folgenden zunächst näher erläutert werden.

Wohl der Allgemeinheit und im Einklang mit ihm auch dem Nutzen Einzelner dienen und dass jede vermeidbare Beeinträchtigung unterbleibt. Wohl aus diesen Gründen sind die Statistiken der Wasserwirtschaft die ältesten der hier betrachteten. Nach heutigem Stand umfasst das Umweltstatistik-
Strohs zur Energieerzeugung in einem dezentralen Biomasseheizkraftwerk und den Einsatz verarbeiteten, selbst produzierten Rapses als Biodiesel für den betriebseigenen Fuhrpark.

Für eine systematische und kontinuierliche Identifizierung und Prüfung derartiger ökonomischökologischer Optimierungsmaßnahmen wird für die Agrargenossenschaft momentan ein unternehmensspezifisches SEFR-System entwickelt. Die Basis bildet eine Excel-Tabellenkalkulation, die bei Bedarf Ausgangspunkt für eine Access-Datenbank sein kann. Die Microsoft-Produktpalette ist - wie in vielen kleinen und mittelständischen Unternehmen - in der Agrargenossenschaft vorhanden und erfordert folglich geringen Einarbeitungsaufwand.

\section{Anmerkungen}

(1) Basis hierfür war eine systematische Vorstudie. Vgl. Kramer, M./ Brauweiler, J./ Lehmann, M.: Branchenstrukturanalyse: Deutschland, Polen, Tschechien und Euroregion Neisse. In: Osteuropa-Wirtschaft, Heft 3/2000, S. 221-256.

(2) Vgl. Kreeb, M.: Umweltkostenmanagement für mittelständische Unternehmen. Diskussionspapier-Heft 23, Universität Witten/Herdecke 1999.

\section{Die Autorlnnen}

Prof. Dr. Matthias Kramer ist Leiter des Studiengangs Betriebswirtschaftslehre im Internationalen Hochschulinstitut Zittau. Hans-Christian Brauweiler und Peggy Eifler sind dort wiss. Mitarbeiterlnnen.

Kontakt: IHI Zittau, Markt 23, 02763 Zittau,

Tel. 03583/ 771-531, Fax -535, eifler@ihi-zittau.de, kramer@ihi-zittau.de,brauweiler_c@ihi-zittau.de

gesetz im Bereich der Wasserwirtschaft 12 Erhebungen, die in fünf Bereiche zusammengefasst werden können (siehe Tab. 1).

\section{Luftverunreinigungen}

Die Erhebungen zur Luftreinhaltung wurden mit dem Umweltstatistikgesetz von 1994 neu eingeführt. Im einzelnen sind dies zwei Erhebungen, nämlich die Erhebung der Luftverunreinigungen und die Erhebung bestimmter ozonschichtschädigender und klimawirksamer Stoffe.

Bei der Erhebung der Luftverunreinigungen wird darauf verzichtet, die betroffenen Betriebe direkt zu befragen. Stattdessen werden die Emissionserklärungen ausgewertet, die von den Anlagenbetreibern an die Gewerbeaufsichtsämter bzw. Landesumweltämter übermittelt wurden.

Die Erhebung bestimmter ozonschichtschädigender und klimawirksamer Stoffe befragt Unternehmen, die entweder bestimmte ozonschichtschädigende Stoffe herstellen, einführen oder ausführen oder ozonschichtschädigende und klimawirksame Stoffe in nennenswertem Umfang zur Herstellung, Instandhaltung oder Reinigung von Erzeugnissen verwenden.

Diese Erhebung dient dazu, quantitativ festzustellen, ob die Produktion und Verwendung der ozonschichtschädigenden Stoffe in der Bundesrepublik abnimmt bzw. völlig eingestellt wird, sowie der Beobachtung der Entwicklung und des Einsatzes von klimawirksamen Ersatzstoffen.

\section{Umweltökonomische Erhebungen}

Im Bereich der umweltökonomischen Statistiken soll die ökonomische Bedeutung des Umweltschutzes quantifiziert werden. Hier sollen 
(c) 20I0 Authors; licensee IÖW and oekom verlag. This is an article distributed under the terms of the Creative Commons Attribution Non-Commercial No Derivates License (http://creativecommons.org/licenses/by-nc-nd/3.o/), which permits unrestricted use, distribution, and reproduction in any medium, provided the original work is properly cited. 